\title{
Perioperative risk factors for delayed extubation after acute type A aortic dissection surgery
}

\author{
Wiriya Maisat ${ }^{1}$, Sasiya Siriratwarangkul ${ }^{1}$, Apiporn Charoensri ${ }^{1}$, Wanchai Wongkornrat ${ }^{2}$, \\ Saowaphak Lapmahapaisan ${ }^{1}$
}

${ }^{1}$ Department of Anesthesiology, ${ }^{2}$ Division of Cardiothoracic surgery, Department of surgery, Faculty of Medicine Siriraj Hospital, Mahidol University, Bangkok, Thailand

Contributions: (I) Conception and design: W Maisat, S Lapmahapaisan; (II) Administrative support: S Lapmahapaisan; (III) Provision of study materials or patients: All authors; (IV) Collection and assembly of data: All authors; (V) Data analysis and interpretation: W Maisat, S Lapmahapaisan; (IV) Manuscript writing: All authors; (VII) Final approval of manuscript: All authors.

Correspondence to: Asst. Prof. Saowaphak Lapmahapaisan. Department of Anesthesiology, Faculty of Medicine Siriraj Hospital, 2 Wanglang Road, Bangkok noi, Bangkok 10700, Thailand. Email: saowaphak.lap@gmail.com

Background: Delayed extubation after cardiac surgery is associated with high morbidity and mortality, increased intensive care unit length of stay, and healthcare cost. Acute type A aortic dissection (ATAAD) generally results in prolonged mechanical ventilation due to the complexity of surgical management and some postoperative complications. This study aimed to elucidate the perioperative risk factors for delayed extubation in patients undergoing ATAAD surgery.

Methods: A retrospective cohort study including 239 patients who were diagnosed with ATAAD and underwent emergency surgery from October 2004 to January 2018 was performed. The potential perioperative risk factors for delayed extubation were collected. This study defined delayed extubation as the time to commence extubation being greater than 48 hours. The clinical data were analyzed with univariate and multivariate analyses to identify risk factors for delayed extubation following ATAAD surgery.

Results: The incidence of delayed extubation was $48.5 \%(\mathrm{n}=116)$. Multiple logistic regression analysis showed perioperative risk factors for delayed extubation included preoperative cardiac tamponade [odds ratio (OR) 3.94, 95\% confidence interval (CI) 1.39-11.17, $\mathrm{P}=0.010$ ], central arterial cannulation (ascending aorta and proximal aortic arch) for cardiopulmonary bypass (CPB) (OR 4.04, 95\% CI: 1.03-15.91, P=0.046), postoperative stroke (OR 10.58, 95\% CI: 2.65-42.25, $\mathrm{P}=0.001$ ), postoperative renal dysfunction that required temporary hemodialysis (OR $6.6095 \% \mathrm{CI}: 1.97-22.11, \mathrm{P}=0.002)$, and re-exploration to stop bleeding (OR 2.65, 95\% CI: 1.00-6.99, P=0.050).

Conclusions: Preoperative cardiac tamponade, central arterial cannulation for CPB, postoperative stroke, postoperative renal dysfunction that required temporary hemodialysis, and re-exploration to stop bleeding are perioperative risk factors for delayed extubation. Identification of the potential risk factors for delayed extubation may help optimize the perioperative management and improve postoperative outcomes of patients undergoing ATAAD surgery.

Keywords: Cardiopulmonary bypass; extubation; acute type A aortic dissection (ATAAD); risk factors

Submitted Feb 03, 2020. Accepted for publication Jul 28, 2020.

doi: $10.21037 /$ jtd-20-742

View this article at: http://dx.doi.org/10.21037/jtd-20-742 


\section{Introduction}

Acute type A aortic dissection (ATAAD) is defined as the dissection that arises within the ascending aorta or aortic arch, according to Stanford's classification, and occurs less than 14 days from the onset of symptoms (1). This condition is considered a surgical emergency because the mortality rate increases by approximately $1 \%$ for every hour of delay in surgical intervention. ATAAD is associated with high morbidity and mortality, given the surgical inhospital mortality rate of $6.6-23.9 \%$ (2-7). Perioperative management of ATAAD is challenging due to urgent presentation, high risk of mortality, organ dysfunction, and the complexity of surgical management.

Postoperative mechanical ventilation is routinely used after cardiac surgery to minimize myocardial oxygen consumption and work of breathing from spontaneous ventilation. Nevertheless, delayed extubation may provoke adverse effects, including cardiopulmonary compromise, increased risk of pulmonary infection, patient discomfort from the endotracheal tube, increased intensive care unit (ICU) length of stay (LOS), and increased healthcare cost $(8,9)$. Recently, fast track cardiac anesthesia has been introduced into clinical practice and has become increasingly adopted in many institutions. Early extubation within 6-8 hours after some certain cardiac surgeries can reduce the ICU LOS and healthcare costs $(5,8,10)$. Moreover, it also increases the number of cardiac surgeries that can be performed (11). Unfortunately, early tracheal extubation after ATAAD surgery may not be feasible because of postoperative respiratory and ventricular dysfunctions following prolonged cardiopulmonary bypass (CPB), hemodynamic instability, abnormal hemostasis, hypothermia, bleeding, and postoperative complications. However, prolonged mechanical ventilation is associated with increased morbidity, mortality, and nosocomial infection (11). Pierre et al. reported a five-fold increase in mortality in patients with prolonged intubation time following cardiac surgery (12). Even though there is a reasonable amount of past literature available that reports the risk factors for delayed extubation after cardiac surgery, few studies particularly focused on patients who underwent ATAAD surgery $(5,6,13)$. Therefore, this study aimed to elucidate the perioperative risk factors for delayed extubation in patients undergoing ATAAD surgery.

\section{Methods}

This retrospective cohort study was carried out at a tertiary university hospital from October 2004 to January 2018. The study was conducted in accordance with the Declaration of Helsinki (as revised in 2013). The Institutional Review Board (IRB) approved this study and the requirement for individual consent was waived (Si 159/2017). The study criteria included patients diagnosed with ATAAD who underwent emergency surgery. We excluded patients who had preoperative tracheostomy, or who died intraoperatively or within 48 hours after the operation. The medical records of 239 patients were obtained from the hospital electronic database.

All patients were admitted to the surgical cardiac ICU after surgery. Intermittent intravenous morphine was given as a standard analgesia protocol without other sedative medications during mechanical ventilation. A standardized weaning protocol was applied to all patients. Tracheal extubation was performed when patients were fully awake with adequate muscle strength and had normal temperature, stable hemodynamic, acceptable hemostasis, and adequate gas exchange and respiratory mechanics.

\section{Data collection and definition}

Delayed extubation was defined as the cumulative duration of mechanical ventilation of more than 48 hours postoperatively, starting from the cardiac ICU admission to the time in which patients were tracheally extubated. Patients who had an unsuccessful attempt of extubation with an accumulated duration of mechanical ventilation of at least 48 hours were also included. However, there is no standardized definition of delayed extubation following ATAAD surgery. Therefore, we adopted this current definition according to previously conducted studies $(5,6,13)$ and our institutional experience.

Potential perioperative risk factors for delayed extubation were recorded. Preoperative factors included patient characteristics and preoperative complications that were related to ATAAD (cardiac tamponade, coronary involvement, stroke, limb ischemia, visceral ischemia, aortic regurgitation, tracheal intubation, and cardiac arrest). Intraoperative factors included types of surgery, numbers of operation, operative time, sites of arterial cannulation, $\mathrm{CPB}$ and aortic cross-clamp times, as well as deep hypothermic circulatory arrest (DHCA) time. Postoperative factors included stroke, renal dysfunction requiring temporary hemodialysis, re-exploration to stop bleeding, and acute respiratory distress (ARDS). Other postoperative outcomes, namely, mechanical ventilation time, the incidence of 


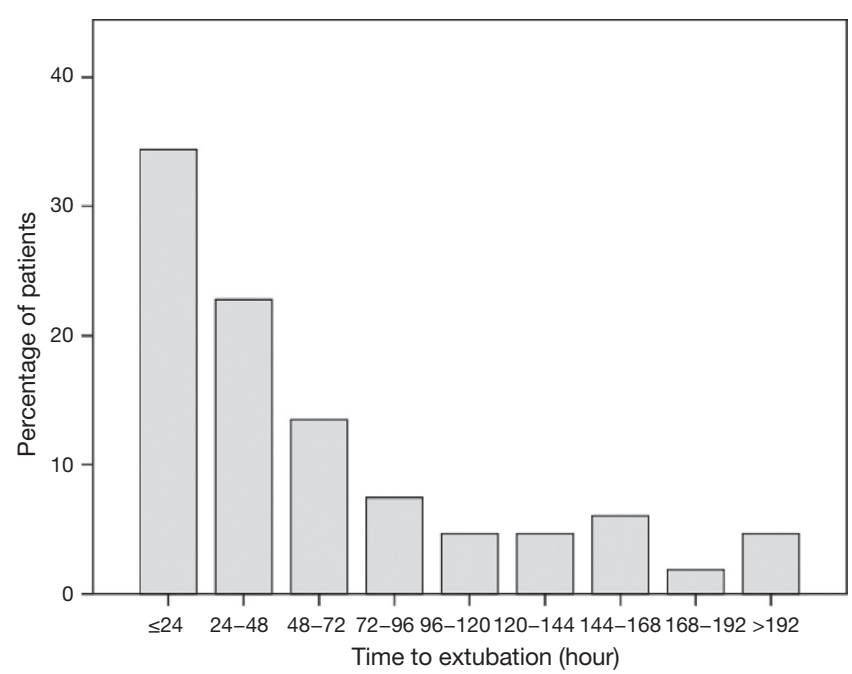

Figure 1 Distribution of extubation time following ATAAD surgery.

tracheostomy, ICU and hospital LOS, and mortality were also recorded.

\section{Statistical analysis}

The statistical analysis was performed using PASW Statistics for Windows, Version 18.0 (SPSS Inc., Chicago, IL, USA). A P value of $<0.05$ was considered statistically significant. Data were presented as frequency and percent values for categorical variables. Mean and standard deviation (SD) was determined for continuous variables with normal distributions; median and interquartile ranges were used for skewed distributions.

Potential perioperative risk factors for delayed extubation were initially compared by univariate analysis using independent sample $t$-test or Mann Whitney $\mathrm{U}$ test for continuous variables and Chi-square or Fisher's exact test for categorical variables. Each variable with a statistically significant difference $(\mathrm{P}<0.05)$ by univariate analysis was entered into multiple logistic regression models.

A multivariate binary logistic regression, using a stepwise approach, was used to identify independent risk factors for delayed extubation. All continuous variables were transformed into categorical variables before the analysis. The cut-off was considered as the $50^{\text {th }}$ percentile of the recorded values and clinically appropriate. Adjusted odd ratio $(\mathrm{OR})$ and $95 \%$ confidence interval $(95 \% \mathrm{CI})$ were used to determine the strength of the association.

Collinearity between variables was assessed by Pearson pairwise correlation coefficient and variance inflation factors (VIF) statistics; $r>0.7$ and VIF $>0.5$ are cut-off values for multicollinearity in the regression model (14). Consequently, the factors that indicated multicollinearity were removed from the model. The Hosmer-Lemeshow goodness-of-fit test was used to assess the validity of the regression model. Multiple imputation was performed with Markov chain Monte Carlo technique in order to alleviate the effect of missing data for variables in multivariate analysis.

\section{Results}

A total of 239 patients were enrolled in this study over a period of 15 years. The patients were divided into early extubation group $(\mathrm{n}=123,51.5 \%)$ and delayed extubation group ( $\mathrm{n}=116,48.5 \%)$. The median extubation time was 34.7 hours, with a range of 6 to 398 hours. The distribution of extubation time had a highly skewed pattern and was illustrated in Figure 1.

A number of perioperative factors were associated with delayed extubation in univariate analysis. The preoperative risk factors were age, preoperative tracheal intubation, and cardiac tamponade (Table 1). The indications for preoperative tracheal intubation were cardiogenic shock from acute myocardial infarction, acute pulmonary edema from severe acute aortic regurgitation, and sudden cardiac arrest.

All of the operations were performed as emergency operations. The operative techniques are summarized in Table 2. Over $50 \%$ of patients required combined surgical procedures of at least two procedures in the same setting. Simultaneous coronary artery bypass graft (CABG) surgery was performed in $10 \%$ of patients. Intraoperative factors that were associated with delayed extubation were total arch replacement surgery, aortic valve surgery, operative time, CPB time, aortic cross-clamp time, and site of arterial cannulation (Table 2). Postoperative stroke, renal dysfunction requiring temporary hemodialysis, and reexploration to stop bleeding were postoperative factors associated with delayed extubation (Table 3).

Multivariate analysis revealed cardiac tamponade, arterial cannulation at ascending aorta and proximal aortic arch, postoperative stroke, postoperative renal dysfunction requiring temporary hemodialysis, and re-exploration to stop bleeding as independent risk factors for delayed extubation following ATAAD surgery (Table 4).

Postoperative outcomes are presented in Table 5. 
Table 1 Univariate analysis of preoperative factors for delayed extubation

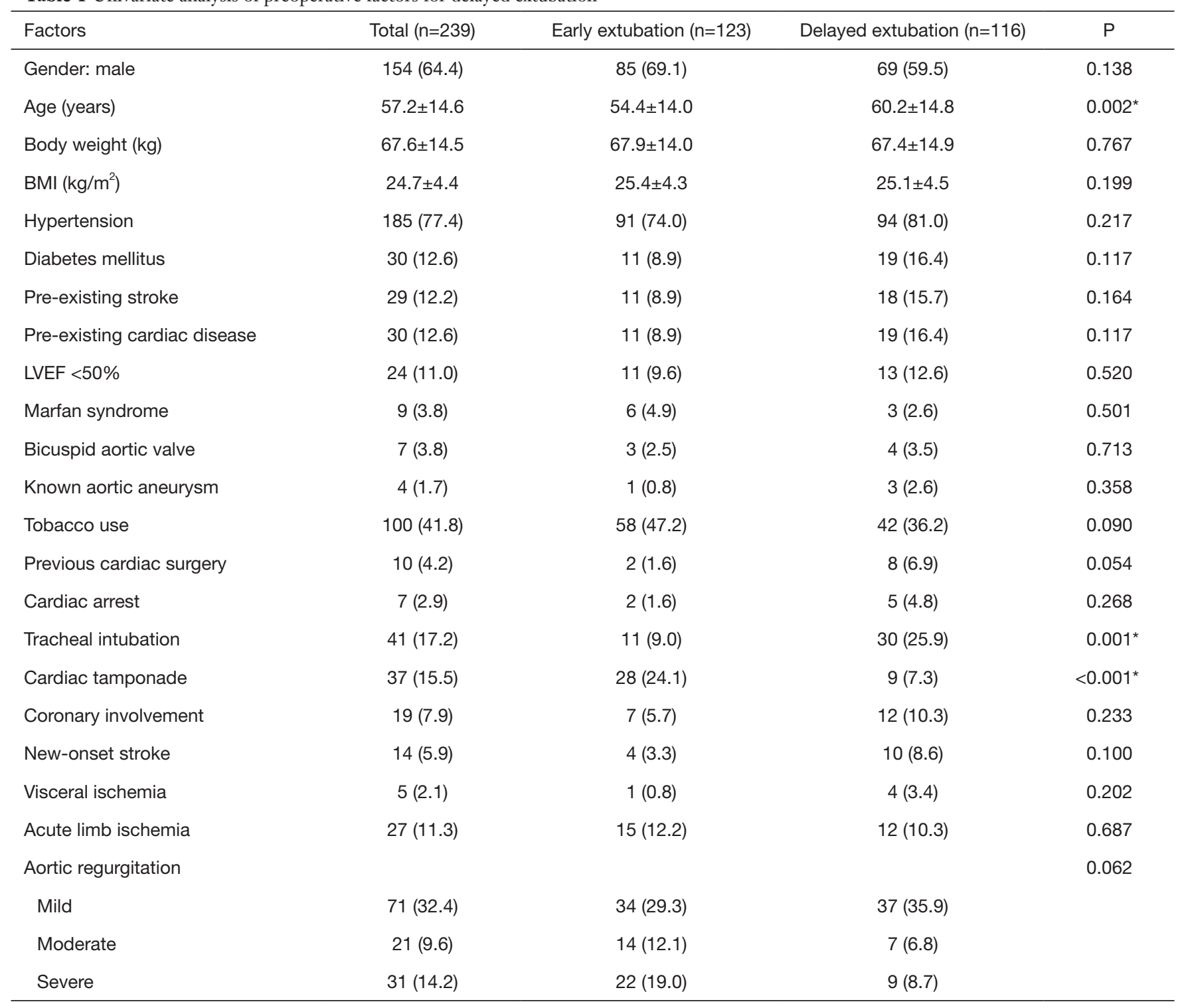

${ }^{*}$, statistical significance. Data were presented as $\mathrm{n}(\%)$ or mean \pm SD. LVEF, left ventricular ejection fraction.

Tracheostomy was performed in 16 patients $(4.7 \%)$ who could not be extubated at 6-22 days postoperatively. The timing of the tracheostomy was at the discretion of the attending cardiac surgeons. There was no incidence of deep sternal wound infection in any of the patients who had tracheostomy. The ICU and hospital LOS were significantly longer in delayed extubation group. The overall incidence of in-hospital mortality was $10.0 \%(n=24)$. The in-hospital mortality was observed only in patients who had delayed extubation.

\section{Discussion}

This study reported perioperative risk factors for delayed extubation following ATAAD surgery. We demonstrated that preoperative cardiac tamponade, ascending aorta, and proximal aortic arch as arterial cannulation (central arterial cannulation) for $\mathrm{CPB}$, postoperative stroke, postoperative renal dysfunction requiring temporary hemodialysis, and reexploration to stop bleeding were independent risk factors for delayed extubation following ATAAD surgery. 
Table 2 Univariate analysis of intraoperative factors for delayed extubation

\begin{tabular}{|c|c|c|c|c|}
\hline Factors & Total $(n=239)$ & Early extubation $(n=123)$ & Delayed extubation $(n=116)$ & $P$ \\
\hline Ascending aorta replacement & 191 (79.9) & $99(80.5)$ & $92(79.3)$ & 0.872 \\
\hline Hemiarch replacement & $78(32.6)$ & $39(31.7)$ & $39(33.6)$ & 0.784 \\
\hline Total arch replacement & $48(20.1)$ & $16(13.0)$ & $32(27.6)$ & $0.006^{*}$ \\
\hline Aortic valve surgery & $22(9.2)$ & $16(13.0)$ & $6(5.2)$ & $0.044^{*}$ \\
\hline CABG & $24(10.0)$ & $10(8.1)$ & $14(12.1)$ & 0.390 \\
\hline Numbers of operation & & & & 0.102 \\
\hline 1 & $97(40.6)$ & $58(47.3)$ & $39(33.6)$ & \\
\hline 4 & $8(3.8)$ & $4(3.3)$ & $4(3.4)$ & \\
\hline Operative time (min) & $419.4 \pm 214.8$ & $378.9 \pm 206.2$ & $462.4 \pm 216.3$ & $0.003^{*}$ \\
\hline CPB time (min) & $182.6 \pm 98.3$ & $161.23 \pm 71.7$ & $205.2 \pm 116.3$ & $<0.001^{\star}$ \\
\hline Aortic cross-clamp time (min) & $133.5 \pm 72.9$ & $118 \pm 62.6$ & $149.9 \pm 79.3$ & $0.001^{*}$ \\
\hline DHCA time (min) & $36.4 \pm 32.3$ & $34.2 \pm 27.8$ & $38.9 \pm 36.5$ & 0.260 \\
\hline Arterial cannulate site & & & & $0.002^{*}$ \\
\hline Axillary & $99(42.3)$ & $62(50.8)$ & $37(33.0)$ & \\
\hline Central & $21(9.0)$ & $8(6.6)$ & $13(11.6)$ & \\
\hline ACP & 175 (73.2) & $89(73.0)$ & $86(74.1)$ & \\
\hline $\mathrm{RCP}$ & $22(9.2)$ & $14(11.5)$ & $8(6.9)$ & \\
\hline Combined ACP and RCP & $5(2.1)$ & $3(2.5)$ & $2(1.7)$ & \\
\hline None & $36(15.1)$ & $16(13.1)$ & $20(17.2)$ & \\
\hline
\end{tabular}

*, statistical significance. Data were presented as $\mathrm{n}(\%)$ or mean \pm SD. ACP, antegrade cerebral perfusion; CPB, cardiopulmonary bypass; DHCA, deep hypothermic circulatory arrest; RCP, retrograde cerebral perfusion.

Most of the previous studies used 48 hours as the cutoff values for delayed extubation after ATAAD surgery postoperatively $(5,6,13)$. The reported incidence of delayed extubation ranged from $28.9 \%$ to $36.1 \%(5,6,13)$. This proportion is noticeably lower than that of our study $(48.5 \%)$. The lowest incidence of delayed extubation was demonstrated in the study by Jin et al. (5). They retrospectively included 121 patients who underwent total arch replacement with frozen elephant trunk in 2013-2014. Conversely, Kimura et al. presented the highest number $(36.1 \%)$ of delayed extubation (13). Their study was conducted in 233 patients who underwent emergency ATAAD surgery from 1996 to 2007. The extent of surgery was quite similar to ours. The incidence of postoperative 
Table 3 Univariate analysis of postoperative factors for delayed extubation

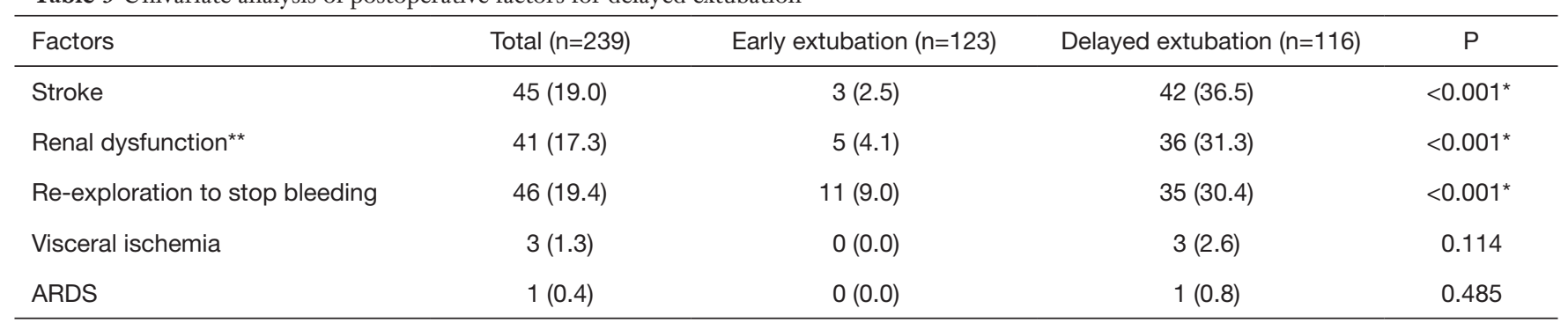

${ }^{*}$, statistical significance; ${ }^{*}$, renal dysfunction requiring temporary hemodialysis. Data were presented as $\mathrm{n}(\%)$. ARDS, acute respiratory distress syndrome.

Table 4 Multivariate analysis of perioperative factors for delayed extubation following ATAAD surgery

\begin{tabular}{|c|c|c|}
\hline Factors & Adjust OR (95\% Cl) & $\mathrm{P}$ \\
\hline \multicolumn{3}{|l|}{ Preoperative factors } \\
\hline Age $>65$ years & $2.01(1.00-4.39)$ & 0.051 \\
\hline Tracheal intubation & $1.41(0.51-3.90)$ & 0.531 \\
\hline Cardiac tamponade & $3.94(1.39-11.17)$ & $0.010^{*}$ \\
\hline \multicolumn{3}{|l|}{ Intraoperative factors } \\
\hline Total arch replacement & $1.46(0.59-3.59)$ & 0.414 \\
\hline Aortic valve surgery & $0.30(0.08-1.12)$ & 0.072 \\
\hline Operative time $>7$ hours & $2.31(0.97-5.49)$ & 0.058 \\
\hline CPB time $>3$ hours & $1.96(0.88-4.35)$ & 0.101 \\
\hline \multicolumn{3}{|l|}{ Arterial cannulation site } \\
\hline Axillary & 1 (reference) & - \\
\hline Central & $4.04(1.03-15.91)$ & $0.046^{*}$ \\
\hline Innominate & $0.66(0.17-2.52)$ & 0.543 \\
\hline Femoral & $1.11(0.44-2.78)$ & 0.830 \\
\hline Multiple & $4.37(0.40-48.36)$ & 0.229 \\
\hline \multicolumn{3}{|l|}{ Postoperative factors } \\
\hline Stroke & $10.58(2.65-42.25)$ & $0.001^{*}$ \\
\hline Renal dysfunction ${ }^{\star *}$ & $6.60(1.97-22.11)$ & $0.002^{*}$ \\
\hline $\begin{array}{l}\text { Re-exploration to stop } \\
\text { bleeding }\end{array}$ & 2.65 (1.00-6.99) & $0.050^{*}$ \\
\hline
\end{tabular}

${ }^{*}$, statistical significance; ${ }^{\star}$, renal dysfunction requiring temporary hemodialysis. CPB, cardiopulmonary bypass.

stroke, postoperative temporary hemodialysis, and reexploration to stop bleeding in our study is approximately 2-4 times greater than that of the aforementioned studies
$(5,13)$. These complications significantly predicted delayed extubation in this study, and this might explain the high incidence of delayed extubation. Another confounding factor that contributed to delayed extubation was that none of the patients was extubated between $11 \mathrm{pm}$ and 6 am due to the limited number of attending doctors and nurses.

The predictive factors for prolonged mechanical ventilation following ATAAD surgery were inconsistent among preceding studies; these include preoperative shock, preoperative limb ischemia, postoperative renal failure (serum creatinine $>2 \mathrm{mg} / \mathrm{dL}$ ), concomitant CABG, low preoperative platelet count, high serum lactate level, increased age, and high preoperative leukocyte count $(5,6,13)$. We found that preoperative cardiac tamponade was a significant predictor for delayed extubation after ATAAD surgery. Rupture of the ascending aorta or the extension of the dissection into aortic root can produce hemopericardium and cardiac tamponade $(15,16)$. Preoperative cardiac tamponade might contribute to delayed extubation; according to Bayegan et al., several patients suffered postoperative critical illness that resulted in multi-organ failure (15). They also revealed that patients with preoperative severe cardiac tamponade had a 16-fold increased risk of multi-organ failure and in-hospital death.

Site of arterial cannulation for $\mathrm{CPB}$ was the only intraoperative factor associated with delayed extubation from a multivariate analysis. Central arterial cannulation for CPB was a significant predictor for delayed extubation in our study. According to the study by Rosinski et al., central cannulation was associated with a higher occurrence of stroke because it could potentiate the propagation of clot or embolization (17). As a result, it could be implied that patients might require prolonged mechanical ventilation due to postoperative stroke. 
Table 5 Postoperative outcomes

\begin{tabular}{|c|c|c|c|c|}
\hline Outcomes & Total $(n=239)$ & Early extubation $(n=123)$ & Delayed extubation $(n=116)$ & $\mathrm{P}$ \\
\hline Tracheostomy & $16(4.7)$ & - & $16(4.7)$ & - \\
\hline ICU LOS (day) & $4.0(3.0,7.0)$ & $3.0(2.0,4.0)$ & $7.0(5.0,12.5)$ & $<0.001^{\star}$ \\
\hline Hospital LOS (day) & $11(8.0,17.0)$ & $8.0(7.0,11.0)$ & $15.0(10.0,25.0)$ & $<0.001^{\star}$ \\
\hline
\end{tabular}

*, statistical significance. Data were presented as n (\%), or median (IQR). LOS, length of stay; ICU, intensive care unit.

Prolonged operative time and $\mathrm{CPB}$ time were generally considered as risk factors for delayed extubation. Because prolonged $\mathrm{CPB}$, in combination with intraoperative DHCA, hemorrhage, and massive transfusion, can activate the inflammatory cascade and further result in respiratory compromise (18). However, they were statistically significant factors as shown by univariate analysis but were not significant in multivariate analysis. This finding was consistent with previously published studies $(5,13)$.

Postoperative complications are strong independent predictors for delayed extubation. Postoperative stroke was one of the risk factors with an incidence of $19 \%$. This proportion is relatively higher than the previously reported incidence of $3-10 \%(5,13,19)$. Cerebral tissue hypoperfusion and emboli during $\mathrm{CPB}$, as well as direct compromise of cerebral circulation are the possible mechanisms of stroke after ATAAD surgery.

Another postoperative predictor of delayed extubation was renal dysfunction that required temporary hemodialysis. Kimura $e t a l$. also identified postoperative renal failure (defined as creatinine $>2.0 \mathrm{mg} / \mathrm{dL}$ ) as an independent risk factor for delayed extubation following ATAAD surgery (13). These findings might imply that cerebral and renal protective strategies should be optimized in order to minimize the incidence of delayed extubation after ATAAD surgery and its consequences afterward. Further, reexploration to stop bleeding was another postoperative factor for delayed extubation. Postoperative bleeding that requires surgery is usually associated with unstable hemodynamics, which is not compatible with the extubation criteria.

This study had some limitations. First, some relevant data were missing because of the nature of retrospective study designs and the emergency treatment of ATAAD. For example, massive blood transfusion was evidently related to prolonged mechanical ventilation $(20,21)$; unfortunately, we were unable to assess the exact volume of blood transfusion due to poorly collected data. Furthermore, the data of this study were collected from a single tertiary university hospital. The variation among institutions, in terms of anesthesia and surgical techniques, including the quality of postoperative care, may affect extubation outcomes. Because we included all patients who had ATAAD surgery consecutively during the study period, the onset of aortic dissection varied among individuals. The longer onset of symptoms is acknowledged as one of the risk factors for mortality after ATAAD; therefore, it might have affected the extubation time.

\section{Conclusions}

Preoperative cardiac tamponade, central arterial cannulation for $\mathrm{CPB}$, postoperative stroke, postoperative renal dysfunction requiring temporary hemodialysis, and re-exploration to stop bleeding are independent perioperative risk factors for delayed extubation. Delayed extubation is significantly associated with increased in-hospital mortality and increased ICU and hospital LOS. Identification of the risk factors for delayed extubation is beneficial to optimize perioperative management of patients undergoing ATAAD surgery.

\section{Acknowledgments}

Funding: This study was supported by a research grant from Faculty of Medicine Siriraj Hospital, Mahidol University, Thailand.

\section{Footnote}

Data Sharing Statement: Available at http://dx.doi. org/10.21037/jtd-20-742

Conflicts of Interest: All authors have completed the ICMJE 
uniform disclosure form (available at http://dx.doi. org/10.21037/jtd-20-742). The authors have no conflicts of interest to declare.

Ethical Statement: The authors are accountable for all aspects of the work in ensuring that questions related to the accuracy or integrity of any part of the work are appropriately investigated and resolved. The study was conducted in accordance with the Declaration of Helsinki (as revised in 2013). This study was approved by Siriraj Institutional Review Board with the exemption of individual consent (Si 159/2017).

Open Access Statement: This is an Open Access article distributed in accordance with the Creative Commons Attribution-NonCommercial-NoDerivs 4.0 International License (CC BY-NC-ND 4.0), which permits the noncommercial replication and distribution of the article with the strict proviso that no changes or edits are made and the original work is properly cited (including links to both the formal publication through the relevant DOI and the license). See: https://creativecommons.org/licenses/by-nc-nd/4.0/.

\section{References}

1. Hirst AE, Johns VJ, Kime SW. Dissecting aneurysm of the aorta: a review of 505 cases. Medicine 1958;37:217-79.

2. Pape LA, Awais M, Woznicki EM, et al. Presentation, diagnosis, and outcomes of acute aortic dissection: 17-year trends from the International Registry of Acute Aortic Dissection. J Am Coll Cardiol 2015;66:350-8.

3. Rampoldi V, Trimarchi S, Eagle KA, et al. Simple risk models to predict surgical mortality in acute type A aortic dissection: the International Registry of Acute Aortic Dissection score. Ann Thorac Surg 2007;83:55-61.

4. Smith HN, Boodhwani M, Ouzounian M, et al. Classification and outcomes of extended arch repair for acute Type A aortic dissection: a systematic review and meta-analysis. Interact Cardiovasc Thorac Surg 2017;24:450-9.

5. Jin $M, M a W G$, Liu S, et al. Predictors of prolonged mechanical ventilation in adults after acute type-A aortic dissection repair. J Cardiothorac Vasc Anesth 2017;31:1580-7.

6. Li CN, Chen L, Ge YP, et al. Risk factors for prolonged mechanical ventilation after total aortic arch replacement for acute DeBakey type I aortic dissection. Heart Lung
Circ 2014;23:869-74.

7. Wen $\mathrm{M}, \mathrm{Han} \mathrm{Y}, \mathrm{Ye} \mathrm{J}$, et al. Peri-operative risk factors for in-hospital mortality in acute type A aortic dissection. J Thorac Dis 2019;11:3887-95.

8. Cislaghi F, Condemi AM, Corona A. Predictors of prolonged mechanical ventilation in a cohort of 5123 cardiac surgical patients. Eur J Anaesthesiol 2009;26:396-403.

9. Prakash O, Jonson B, Meij S, et al. Criteria for early extubation after intracardiac surgery in adults. Anesth Analg 1977;56:703-8.

10. Cheng DC, Karski J, Peniston C, et al. Early Tracheal Extubation after Coronary Artery Bypass Graft Surgery Reduces Costs and Improves Resource UseA Prospective, Randomized, Controlled Trial. Anesthesiology 1996;85:1300-10.

11. Hawkes CA, Dhileepan S, Foxcroft DR. Early extubation for adult cardiac surgical patients. Cochrane Database Syst Rev 2003;(4):CD003587.

12. Suarez-Pierre A, Fraser CD, Zhou X, et al. Predictors of operative mortality among cardiac surgery patients with prolonged ventilation. J Card Surg 2019;34:759-66.

13. Kimura N, Tanaka M, Kawahito K, et al. Risk factors for prolonged mechanical ventilation following surgery for acute type A aortic dissection. Circ J 2008;72:1751-7.

14. Vatcheva KP, Lee M, McCormick JB, et al. Multicollinearity in regression analyses conducted in epidemiologic studies. Epidemiology (Sunnyvale). 2016;6:227.

15. Bayegan K, Domanovits H, Schillinger M, et al. Acute type A aortic dissection: the prognostic impact of preoperative cardiac tamponade. Eur J Cardiothorac Surg 2001;20:1194-8.

16. Isselbacher EM, Cigarroa JE, Eagle KA. Cardiac tamponade complicating proximal aortic dissection. Is pericardiocentesis harmful? Circulation 1994;90:2375-8.

17. Rosinski BF, Idrees JJ, Roselli EE, et al. Cannulation strategies in acute type A dissection repair: a systematic axillary artery approach. J Thorac Cardiovasc Surg 2019;158:647-59. e5.

18. Nakajima T, Kawazoe K, Izumoto H, et al. Risk factors for hypoxemia after surgery for acute type A aortic dissection. Surg Today 2006;36:680-5.

19. Bavaria JE, Brinster DR, Gorman RC, et al. Advances in the treatment of acute type A dissection: an integrated approach. Ann Thorac Surg 2002;74:S1848-52.

20. Habib RH, Zacharias A, Engoren M. Determinants of 
prolonged mechanical ventilation after coronary artery bypass grafting. Ann Thorac Surg 1996;62:1164-71.

21. Suematsu Y, Sato H, Ohtsuka T, et al. Predictive risk factors for delayed extubation in patients undergoing coronary artery bypass grafting. Heart Vessels 2000;15:214-20.

Cite this article as: Maisat W, Siriratwarangkul S, Charoensri A, Wongkornrat W, Lapmahapaisan S. Perioperative risk factors for delayed extubation after acute type A aortic dissection surgery. J Thorac Dis 2020;12(9):4796-4804. doi: 10.21037/jtd-20742 